\title{
CONTAMINATION OF GOWNS AND DEVICES USED BY HOSPITAL STAFF
}

\author{
Ü. Parm ${ }^{1}$, K. Paal ${ }^{1}$, K. Poren ${ }^{2}$ \\ ${ }^{1}$ Tartu Health Care College, Tartu, ${ }^{2}$ North Estonia Medical Centre Foundation, Tallinn, Estonia
}

\begin{abstract}
Aim: To determine the microbial contamination by Gram-negative and Gram-positive opportunistic microbes of nurses' gowns and devices that hospital staff uses in order to assess the risk of transmission of possible hospital infection agents.
\end{abstract}

Methods: A total of 23 nurses from different pediatric units of Tartu Clinics, Health Care Centre and Health Care College were involved in June 2010. Samples were taken from three different sites of gowns, cell phones, pens, necklaces or neckbands, and nameplates. The plating to McConkey, blood agar and later Etests were applied.

Results: Microbial growth was observed in 137/184 (74.5\%). In 60 samples more than one microbe species were isolated (in maximum of 4). A total of 203 Gram-positive and 25 Gram-negative microbes were isolated. Frequent Gram-positives were Micrococcus spp (25.1\%), S. epidermidis (21.2\%), and S. xylosus $(13.3 \%)$. The edge of pocket and the site of thigh were significant more colonized with different microbes compared with nameplates $(\mathrm{p}=0.007$ and $\mathrm{p}=<0.001$, respectively) and pens ( $\mathrm{p}>0.001$ and $\mathrm{p}=0.011$, respectively). The microbial counts were not dependant on the profile of different units. The possible agents of hospital infections were isolated in intensive care unit mostly from gowns (Enterobacteriaceae $\mathrm{n}=2$; Pseudomonas aeruginosa $\mathrm{n}=3$, Enterococcus $\operatorname{spp} \mathrm{n}=1, S$. aureus $\mathrm{n}=1$ ). Two $S$. aureus isolates were obtained in pediatric unit and one from a student; all were isolated from the edge of pocket.

Conclusions: Gowns may be an important vector for patients-to-patient transmission of agents of hospital infections, especially in intensive care units. 